\title{
9. Développement humain et droits de l'homme
}

\section{Marie Thorndahl, Christoph Stamm et Catherine Schümperli Younossian}

\section{(2)enEdition}

\section{Journals}

Édition électronique

URL : http://journals.openedition.org/aspd/320

DOI : 10.4000/aspd.320

ISSN : 1663-9669

\section{Éditeur}

Institut de hautes études internationales et du développement

\section{Édition imprimée}

Date de publication : 1 avril 2006

Pagination : 137-155

ISBN : 2-88247-061-4

ISSN : 1660-5934

Référence électronique

Marie Thorndahl, Christoph Stamm et Catherine Schümperli Younossian, «9. Développement humain et droits de l'homme », Annuaire suisse de politique de développement [En ligne], 25-1 | 2006, mis en ligne le 12 février 2010, consulté le 08 septembre 2020. URL : http://journals.openedition.org/aspd/ 320 ; DOI : https://doi.org/10.4000/aspd.320 


\section{Développement humain et droits de l'homme*}

\subsection{Sommet mondial sur la société de l'information, phase II, Tunis, $16-18$ novembre 2005}

Le Sommet mondial sur la société de l'information (SMSI), organisé par l'Union internationale des télécommunications (UIT) sous le haut patronage du secrétaire général de l'ONU, Kofi Annan, s'est tenu en deux phases. La première a eu lieu à Genève du 10 au 12 décembre 2003, la seconde à Tunis du 16 au 18 novembre 2005. Cet article dresse un bilan de cette seconde phase, qui avait pour ordre du jour les mécanismes de financement et la gouvernance d'Internet. Pour avoir accueilli la première phase du SMSI, la Suisse était l'hôte d'honneur de Tunis où elle s'est démarquée en déployant une diplomatie particulièrement active dans le domaine des Droits humains.

[DA Annuaire 2004, n 1, SMSI, phase I, Genève, 10-12 décembre 2003, pp. 147-157.

\subsubsection{Objectifs du Sommet de Tunis}

Lors de la première phase du SMSI, les gouvernements ont adopté une Déclaration de principes, qui consigne une vision commune de la société mondiale de l'information («chapeau politique»), ainsi qu'un Plan d'action («partie opérationnelle»), précisant les moyens et mesures nécessaires pour concrétiser cette vision ${ }^{1}$. Le Sommet de Tunis, qui s'est tenu deux ans plus tard, avait pour objectifs :

1) d'assurer le suivi de la Déclaration de principes et du Plan d'action adoptés à Genève, avec une attention particulière pour les pays les moins avancés. Comme l'a appelé de ses vœux le secrétaire général de l'ONU dans son discours d'ouverture, cette seconde phase devait être le «sommet des solutions $»^{2}$;

2) d'examiner et de traduire en documents politiques et en mesures opérationnelles les rapports des groupes d'experts sur les deux questions restées ouvertes à Genève, c'est-à-dire

- le rapport du Groupe de travail sur les mécanismes de financement $(\mathrm{GAMF})^{3}$, chargé de faire un état des lieux et des propositions pour financer les technologies de l'information et de la communication (TIC) dans les régions défavorisées;

* Partie 9.1: par Marie Thorndahl, socio-économiste. Coresponsable du dossier Société de l'information et coopération internationale. development.com de l'Annuaire suisse de politique de développement 2003 (vol. 22, n 2, 2003) et coordinatrice du CD-Rom accompagnant ce dossier. Parties 9.2, 9.3 et 9.5: par Christoph Stamm, politologue.

Partie 9.4: par Catherine Schümperli Younossian, chargée de recherche à l'IUED.

Documents en ligne sur <www.itu.int/wsis $>$.

2 Déclaration de M. Kofi Annan, secrétaire général de l'Organisation des Nations unies, 16 novembre 2005, <www.itu.int/wsis/tunis/statements/docs/io-un-opening/1-fr.html>.

3 Task Force on Financial Mechanisms for ICT for Development, <www.itu.int/wsis/tffm>. 
- le rapport du Groupe de travail sur la gouvernance de l'Internet (GTGI), chargé de traiter des mécanismes de gestion des ressources communes d'Internet comme les noms de domaines et les protocoles techniques.

\subsubsection{Phase préparatoire et documents adoptés}

Trois conférences préparatoires (PrepCom) $\left(1^{\circ}\right.$ Hammamet, Tunisie, juin 2004 ; $2^{\circ}$ Genève, février 2005; $3^{\circ}$ Genève, septembre 2005), six conférences régionales et plus de vingt réunions thématiques faisaient partie du processus préparatoire officiel.

Comme à Genève, les gouvernements ont adopté à Tunis deux documents finaux distincts, soit L'engagement de Tunis («chapeau politique») et l'Agenda de Tunis pour la société de l'information («partie opérationnelle») ${ }^{5}$.

\subsubsection{Participation}

\section{$\square$ Affluence record}

La phase de Tunis a drainé plus de $18^{\prime} 000$ personnes, ce qui en fait la plus grande conférence jamais organisée par les Nations unies. Cette affluence record témoigne d'abord du processus tripartite, les participants non étatiques ayant surpassé en nombre les représentants gouvernementaux.

Ad Annuaire 2003, $\mathrm{n}^{\circ}$ 2, Seán Ó Siochrù et Bruce Girard, «La société civile enlisée dans le système», pp. 207-218.

La société civile était le plus grand groupe, avec 5900 participants. Elle a réussi à avoir une influence notable, notamment en imposant Shirin Ebadi, Prix Nobel de la paix 2003 et militante iranienne des droits humains, comme porte-parole de la société civile à la cérémonie d'ouverture ${ }^{6}$. Les médias avaient quant à eux 1200 représentants. Le secteur privé, qui avait boudé Genève avec quelques centaines de représentants seulement, a montré son influence grandissante dans les affaires internationales, avec une représentation forte de 4000 membres coordonnée à travers la Chambre de commerce internationale.

Cent septante-quatre pays y étaient représentés, à travers 5800 participants. Cependant, sur les 44 chefs d'Etat ou de gouvernement présents au sommet, la plupart venaient d'Afrique - et un seul d'un pays industrialisé, la Suisse. L'absence des chefs d'Etat des principaux pays riches, producteurs et utilisateurs d'Internet, montre un certain désintérêt des «grands» pour cette rencontre.

\section{$\square$ Délégation suisse}

Hôte d'honneur, la Suisse s'est démarquée par sa forte présence à Tunis avec une délégation officielle de 52 membres et ses prises de position remarquées en faveur de la liberté d'expression. Le président de la Confédération, Samuel Schmid, a

\footnotetext{
Working Group on Internet Governance, <www.itu.int/wsis/wgig $>$.

Documents en ligne sur <www.itu.int/wsis $>$.

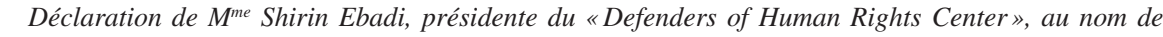
"the Civil Society» International Federation for Human Rights, lors de l'ouverture du SMSI, 16 novembre 2005 , <www.itu.int/wsis/tunis/statements/docs/cs-opening/1-fr.html>.
} 
dirigé la délégation et a tenu le troisième discours ${ }^{7}$ à la cérémonie d'ouverture, après le président tunisien, Zine el-Abidine Ben Ali, et Kofi Annan. Le conseiller fédéral Moritz Leuenberger l'a remplacé ensuite pour participer à une table ronde de haut niveau et représenter la Suisse au cours du débat général ainsi qu'à la cérémonie de clôture. La Suisse était également représentée par Marc Furrer, chef opérationnel de la délégation suisse et président de la Commission fédérale de la communication (COMCOM), et Walter Fust, directeur de la Direction du développement et de la coopération (DDC), ainsi que par des collaborateurs du Département fédéral des affaires étrangères (DFAE), de l'Office fédéral de la communication (OFCOM), de l'Office fédéral de la culture $(\mathrm{OFC})$ et du Secrétariat d'Etat à l'économie (seco).

Contrairement à la phase I, la société civile suisse n'était pas membre de la délégation bien qu'elle ait entretenu un dialogue constant avec les autorités lors du processus préparatoire, notamment à travers comunica-ch, la Coalition suisse pour la société de l'information, qui regroupe une vingtaine d'organisations ${ }^{8}$.

Pour avoir été l'hôte de la première phase du sommet, la Suisse a occupé le pavillon d'honneur, où elle a présenté une exposition réalisée par des étudiants de la Haute Ecole spécialisée des deux Bâles ${ }^{9}$. Cette exposition a été confiée à «Présence Suisse», qui, selon Le Temps, devait permettre à la Suisse de ne pas s'afficher de manière trop évidente aux côtés du régime tunisien ${ }^{10}$. En outre, la Suisse disposait d'un pavillon national dans le cadre de l'exposition ICT4D (Information and Communication Technologies for Development), organisée et financée par la DDC avec un de ses principaux partenaires, le Global Knowledge Partnership (GKP) ${ }^{11}$.

[D] Annuaire 2003, n 2, Gerolf Weigel, «DDC: une approche centrée sur les populations », pp. 135-139.

\subsubsection{Les deux grandes questions à l'ordre du jour}

\section{$\square$ Fracture numérique et financements}

Le premier volet discuté à Tunis concernait la réduction de la «fracture numérique» entre pays riches et pays pauvres. Nombre de médias ont jugé que, en n'y dépêchant pas leurs plus hauts responsables, les pays riches ont entériné de fait leur refus de s'engager sur le principe d'une contribution financière obligatoire pour réduire l'écart technologique entre le Nord et le Sud.

Comme à Genève, les documents adoptés à Tunis réaffirment la nécessité de lutter contre la fracture numérique à travers les partenariats public-privé et l'ouverture des marchés des télécommunications. Ces orientations libérales ont cependant été nuancées dans la Déclaration de Tunis en accordant un rôle plus important aux Etats: ils se sont engagés à mettre les TIC parmi leurs priorités, notamment à travers la mise en place de plans nationaux, les «cyberstratégies», prenant en compte des objectifs sociaux. De Genève à

7 Déclaration de M. Samuel Schmid, président de la Confédération suisse, lors de l'ouverture du SMSI, 16 novembre 2005, <www.itu.int/wsis/tunis/statements/docs/g-switzerland-opening/1-fr.html>.

$8<$ www.comunica-ch.net $>$.

9 Pavillon d'honneur de la Suisse au SMSI, <www.presence.ch/f/500/532.php>.

10 «La Suisse peine à cacher son embarras vis-à-vis de la Tunisie», Le Temps, $1^{\text {er }}$ octobre 2005, $<$ www.letemps.ch/dossiers/dossiersarticle.asp?ID=164615>.

11 ICT for Development Platform, <www.ict-4d.org >. 
Tunis, les textes ont donc légèrement glissé d'un discours du «tout-marché» vers la nécessité de mettre en place des politiques et des investissements publics pour faire des TIC des instruments pour le développement, notamment des régions les plus déshéritées ${ }^{12}$. Au-delà de ces principes, les gouvernements n'ont pas pris d'engagements concrets et n'ont décidé d'aucune option pour suivre les progrès réalisés.

[D Annuaire 2003, no 2, Marie Thorndahl, «Les promesses d'une prospérité virtuelle», pp. 3-26. Annie Chéneau-Loquay et Raphaël Ntambue-Tshimbulu, «La coopération à l'assaut de l'Afrique subsaharienne», pp. 45-75.

Deux initiatives emblématiques ont cependant été saluées par les gouvernements et ont attiré l'attention des médias:

- les Etats se sont félicités - sans pour autant s'y associer - de la création du Fonds mondial pour la solidarité numérique $(\mathrm{FSN})^{13}$, «un mécanisme financier innovant et volontaire ouvert à tous », lancé lors de la première phase du Sommet par les villes de Genève et de Lyon à l'initiative du président sénégalais, Abdoulaye Wade. Trois ans après le lancement de cette idée, le FSN est cependant essentiellement soutenu par des collectivités locales et a récolté 12 millions de francs suisses, ce qui est peu compte tenu des enjeux;

- le projet le plus spectaculaire sur la question de la «fracture numérique» est celui imaginé par le Massachusetts Institute of Technology ${ }^{14}$ : un portable à 100 dollars - surnommé la «machine verte»- pour les enfants des pays défavorisés. Cette prouesse technologique pourrait être commercialisée par les ministères de l'éducation des pays concernés, selon des accords conclus en dehors du processus de négociation officiel.

Ainsi, deux initiatives extérieures au sommet proposent les réponses les plus concrètes à la lutte contre la fracture numérique, évitant ainsi - selon la société civile engagée sur ce dossier - aux gouvernements de mettre en place de nouveaux modèles et ressources pour développer les infrastructures et les contenus dans les pays du Sud ${ }^{15}$.

Da Annuaire 2003, $\mathrm{n}^{\mathrm{o}}$ 2, Marie Thorndahl, «Financements et partage des richesses: des sujets tabous », pp. 201-206.

\section{$\square$ Gouvernance de l'Internet}

Le second enjeu du sommet concernait le dossier apparemment technique mais très politique de la gouvernance de l'Internet. Les Etats-Unis, qui contrôlent de fait la gestion de la toile via l'ICANN (Internet Corporation for Assigned Names and Numbers) et quelques entreprises privées comme Verisign, ont accepté que la communauté internationale s'intéresse à la question. Après une bataille diplomatique assez rude par laquelle les Etats-Unis se sont retrouvés seuls face au reste des nations, un compromis a été adopté par la création d'un Forum international sur la gouvernance d'Internet (FGI) pour poursuivre les discussions au-delà de Tunis. Si nombre de médias dénoncent une «commission

12 Chantal Peyer, SMSI et lutte contre le fossé numérique: la ligne n'a pas pu être établie, veuillez rappeler plus tard!, 30 novembre 2005, <www.comunica-ch.net/article.php3?id_article=274>.

13 Fonds de solidarité numérique, <www.dsf-fsn.org $>$.

14 MIT Media Lab, \$100 Laptop, <http://laptop.media.mit.edu>.

15 «Bien plus aurait pu être réalisé». Déclaration finale de la société civile sur le SMSI, 18 décembre $2005,<w w w . c o m u n i c a-c h . n e t / a r t i c l e . p h p 3 ? i d \_a r t i c l e=279>$. 
alibi » qui entérine le statu quo actuel par lequel les Etats-Unis sortent grands vainqueurs ${ }^{16}$, certains participants au sommet ont bon espoir, en soulignant que ce compromis permet enfin à «tout le monde [d'] être impliqué dans la gouvernance de l'Internet $»^{17}$. «Il s'agit d'un processus. Mais il est important que ces questions ne se décident plus dans un bureau opaque aux Etats-Unis », a affirmé Moritz Leuenberger ${ }^{18}$.

Ainsi, une grande partie de l'Agenda de Tunis pour la société de l'information est consacrée à la gouvernance et rappelle les principes généraux de droit international qui devraient s'appliquer à Internet, ajoutant au débat de nouvelles questions qui ne font pas consensus telles que la protection des données personnelles, les informations illégales, le spam ou encore la protection du consommateur...

[D] Annuaire 2003, $\mathrm{n}^{\circ}$ 2, Stéphane Koch, «Gouvernance de l'Internet: une centralisation du pouvoir inquiétante», pp. 189-194.

\subsubsection{Les droits humains au SMSI}

\section{$\square$ Incidents et médiatisation}

La liberté de la presse et le respect des droits humains n'étaient pas à l'ordre du jour de la phase II puisque ces grands principes avaient déjà été entérinés à Genève. Ils auront malgré tout dominé cette seconde phase du sommet. De nombreux incidents ont jalonné la préparation et le déroulement du SMSI sur le sol tunisien, notamment l'annulation du «Sommet des citoyens ${ }^{19}$, des pressions constantes sur la société civile tunisienne et des interventions policières hors de l'enceinte du SMSI, des agressions et des intimidations de journalistes étrangers et le verrouillage de sites «critiques», notamment $<w w w . i n f o s u d-w s i s . i n f o>$ et $<w w w . s w i s s i n f o . o r g>$, financé par les autorités helvétiques, rendus invisibles à Tunis suite au discours critique de Samuel Schmid. Le fait le plus marquant fut en effet la censure du président de la Confédération sur la télévision nationale tunisienne lors de la retransmission en direct de son discours d'ouverture et dans les traductions distribuées dans la salle de presse. Ces incidents ont fait la une de la presse internationale, reléguant au second plan l'ordre du jour officiel. Ils ont abouti à des protestations sans précédent de la France et des Etats-Unis et à une crise diplomatique entre Tunis et Berne.

\section{$\square$ Tensions entre la Suisse et la Tunisie}

La Suisse s'est profilée comme un défenseur courageux des libertés publiques, selon la presse occidentale. Samuel Schmid, après son discours censuré qui était une première réprimande officielle contre le régime de Ben Ali sur le sol

16 «Tunis ou la mascarade du "machin" onusien», Le Temps, 19 novembre 2005. A Tunis, des promesses et rien d'autre, Radio France Internationale, 19 novembre 2005, <www.rfi.fr/francais/ actu/articles/071/article_39911.asp>.

17 La société civile se joint aux célébrations, après tout, Inter Press Service News Agency, 18 novembre 2005, <www.ipsnews.net>.

18 A Tunis, la Suisse a gagné en prestige, Swissinfo, 18 novembre 2005, <www.swissinfo.org/sfr/ swissinfo.html? siteSect $=105 \&$ sid $=6250514 \& \mathrm{cKey}=1132494128000>$.

19 Citizens' Summit on the Information Society, <www.citizens-summit.org $>$. 
tunisien, est devenu, selon des journalistes, «une sorte de "pop star" pour la société civile tunisienne réprimée... ${ }^{20} \mathrm{ou}$ « un Guillaume Tell de Tunisie ${ }^{21}$.

Cette prise de position a créé de vives tensions entre la Suisse et la Tunisie, durant le sommet et après. Moritz Leuenberger a critiqué les autorités tunisiennes devant les médias internationaux: "La censure du discours de Samuel Schmid est une violation de la liberté d'expression.» Il a aussi dénoncé les conditions dans lesquelles s'est déroulée la conférence de presse qu'il a luimême tenue à Tunis le lendemain de la prestation censurée du président de la Confédération. "Lors de cette conférence de presse, il y avait des personnes qui n'étaient pas des journalistes, conclut Moritz Leuenberger. Ces agents ont attaqué la Suisse comme pays de 1'argent sale. » ${ }^{22}$ Tout de suite après le SMSI, Micheline Calmy-Rey a reçu plusieurs opposants tunisiens au Ministère des affaires étrangères, ce qui a encore ravivé les tensions ${ }^{23}$. La Suisse a donc été un acteur majeur du SMSI. Pays hôte de la phase I, sa diplomatie a été très dynamique durant la phase II dans le domaine des droits humains. La Suisse compte suivre la situation en Tunisie, notamment en cherchant des appuis multilatéraux auprès d'autres pays européens.

\subsubsection{Incertitudes concernant le suivi}

Organisé en deux phases et jalonné par de nombreuses réunions préparatoires, le SMSI a été un processus de négociation qui a duré plus de quatre ans. A sa clôture officielle, de nombreuses incertitudes persistent quant au suivi des engagements et aux structures qui en émergeront.

Malgré les revendications de la société civile internationale qui souhaitait la mise en place d'un mécanisme de suivi sur les engagements en matière de réduction de la fracture numérique, les gouvernements n'ont pas souhaité prendre de décision à ce propos. Ils ont donné mandat au secrétaire général de l'ONU de faire jusqu'à juillet 2006 des propositions au Conseil économique et social des Nations unies (ECOSOC) pour créer ou mandater une structure multipartite de suivi du SMSI. Pour l'instant, l'après-SMSI s'affiche essentiellement sur Internet, à travers diverses initiatives tentant de rassembler les actions dans le domaine, notamment la base de données de projets créée par l'UIT $^{24}$ ou le site WSIS-Online ${ }^{25}$, financé par différents bailleurs de fonds, dont la DDC.

Le Forum sur la gouvernance d'Internet (FGI), qui se réunira pour la première fois courant 2006, n'a pour sa part pas encore de structure claire, le défi étant de mettre en place une organisation permettant la participation des gouvernements, du secteur privé et de la société civile.

20 «Au SMSI, à Tunis, la Suisse ose faire des reproches au régime de Ben Ali», Le Temps, 18 novembre 2005.

21 Samuel Schmid en Guillaume Tell de Tunisie, Swissinfo, 17 novembre 2005, <www.swissinfo. org/sfr/swissinfo.html?siteSect=105\&sid=6247420>.

22 La Suisse proteste auprès de la Tunisie, Swissinfo, 23 novembre 2005, <www.swissinfo.org/sfr/ swissinfo.html?siteSect $=105 \&$ sid $=6262201>$.

23 «La Tunisie dénonce une "attitude inamicale" de la Suisse», Le Temps, 9 décembre 2005.

24 Inventaire des activités du SMSI, <www.itu.int/wsis/stocktaking/scripts/search.asp?lang=fr $>$.

25 Community Platform for the WSIS, <www.wsis-online.net>. 
Finalement, la phase de Tunis a réaffirmé la plupart des grands principes déjà adoptés à Genève. L'absence de nouvelles solutions significatives fait dire à la société civile internationale que «bien plus aurait pu être réalisé» durant ces quatre années de travaux ${ }^{26}$. Il n'en reste pas moins que le processus du SMSI a concouru à deux orientations majeures dans les négociations internationales.

Premièrement, il a contribué à ancrer plus solidement encore le rôle des acteurs non gouvernementaux dans les structures de négociations. Aujourd'hui, il semble difficile de ne plus impliquer des experts et des groupes d'intérêts venus de la société civile et du monde des affaires dans les prises de décisions à venir.

Deuxièmement, la forte mobilisation des mouvements citoyens ainsi que les prises de position suisses ont permis de conjuguer les traditionnelles préoccupations liées aux droits humains aux nouveaux enjeux de la société de l'information. Les textes politiques adoptés affirment l'illégitimité d'un modèle de développement privilégiant la croissance économique et les prouesses technologiques aux dépens des libertés publiques, déclaration qui n'allait pas de soi.

Au niveau suisse, les travaux du SMSI devraient inspirer le remaniement de la stratégie du Conseil fédéral pour la société de l'information ${ }^{27}$. La coalition de la société civile comunica-ch s'inquiète déjà du manque de vision de ce texte et appelle à une large participation de tous les acteurs concernés, pour discuter de ces enjeux au niveau national.

\section{SOURCES}

Déclaration de M. Kofi Annan, secrétaire général de l'Organisation des Nations unies, 16 novembre 2005, <www.itu.int/wsis/tunis/statements/docs/io-un-opening/1-fr.html>.

SMSI, Agenda de Tunis pour la société de l'information, doc. WSIS-05/TUNIS/DOC/6, 25 novembre 2005, <www.itu.int/wsis>.

SMSI, Engagement de Tunis, doc. WSIS-05/TUNIS/DOC/007-F, 15 novembre 2005, <www.itu.int/wsis>.

Conseil fédéral, Stratégie du Conseil fédéral pour une société de l'information en Suisse du 18 février 1998, <http://www.infosociety.ch/site/attachdb/show.asp?id_attach=876>.

Déclaration de M. Samuel Schmid, président de la Confédération suisse, lors de l'ouverture du SMSI, 16 novembre 2005, <www.itu.int/wsis/tunis/statements/docs/g-switzerland-opening/1-fr.html>.

«Bien plus aurait pu être réalisé». Déclaration finale de la société civile sur le SMSI, 18 décembre 2005, $<$ www.comunica-ch.net/article.php3?id_article=279>.

Déclaration de $M^{m e}$ Shirin Ebadi, présidente du "Defenders of Human Rights Center», au nom de "the Civil Society » International Federation for Human Rights, lors de l'ouverture du SMSI, 16 novembre 2005, <www.itu.int/wsis/tunis/statements/docs/cs-opening/1-fr.html>.

MIT Media Lab, $\$ 100$ Laptop, <http://laptop.media.mit.edu>.

Peyer C., «SMSI et lutte contre le fossé numérique: la ligne n’a pas pu être établie, veuillez rappeler plus tard !», 30 novembre 2005, <www.comunica-ch.net/article.php3?id_article=274>.

Le Temps, «La Suisse peine à cacher son embarras vis-à-vis de la Tunisie», $1^{\text {er }}$ octobre 2005 ; «Au SMSI, à Tunis, la Suisse ose faire des reproches au régime de Ben Ali», 18 novembre 2005; «Tunis ou la mascarade du "machin" onusien», 19 novembre 2005 ; "La Tunisie dénonce une "attitude inamicale" de la Suisse», 9 décembre 2005.

Radio France Internationale, A Tunis, des promesses et rien d'autre, 19 novembre 2005.

Inter Press Service News Agency, La société civile se joint aux célébrations, après tout, 18 novembre 2005.

Swissinfo, Samuel Schmid en Guillaume Tell de Tunisie, 17 novembre 2005; A Tunis, la Suisse a gagné en prestige, 18 novembre 2005; La Suisse proteste auprès de la Tunisie, 23 novembre 2005.

26 «Bien plus aurait pu être réalisé... », op. cit.

27 Conseil fédéral, Stratégie du Conseil fédéral pour une société de l'information en Suisse du 18 février 1998, <http://www.infosociety.ch/site/attachdb/show.asp?id_attach=876>. 


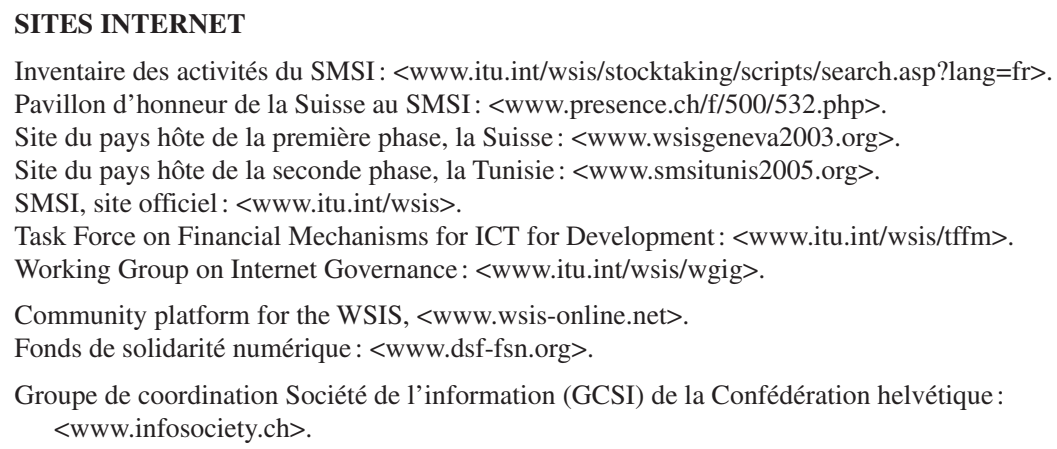

Citizens' Summit on the Information Society: <www.citizens-summit.org $>$. Coalition suisse pour la société de l'information: <www.comunica-ch.net>. ICT for Development Platform : <www.ict-4d.org $>$.

\subsection{Assemblée mondiale de la santé et sujets d'actualité à l'OMS}

La $58^{e}$ session de l'Assemblée mondiale de la santé s'est tenue à Genève du 16 au 26 mai $2005^{28}$ et s'est principalement consacrée à la révision et à l'adoption du Règlement sanitaire international (RSI). Elle a par ailleurs adopté des résolutions dans les domaines suivants: prévention et lutte contre les maladies contagieuses, nutrition chez le nourrisson et le jeune enfant, interventions sanitaires en cas de crise et de catastrophe, usage nocif de l'alcool, prévention et lutte anticancéreuses, grippe et paludisme.

Les Etats membres de l'Organisation mondiale de la santé (OMS) ont décidé, en 1995 déjà, de réviser le Règlement sanitaire international afin d'améliorer les moyens de lutte contre la propagation de maladies contagieuses à l'échelle mondiale. L'épidémie de SRAS (syndrome respiratoire aigu sévère), en 2003, a accéléré ce processus. La nouvelle version du règlement a pour objectif d'empêcher la propagation internationale de maladies par une identification précoce d'événements susceptibles de représenter un danger pour la santé publique.

Les délégués des 192 Etats membres ont approuvé le budget de programme pour 2006 et 2007, dont le total se monte à 3,3 milliards de dollars. Le budget ordinaire s'accroît ainsi de $4 \%$ et les contributions volontaires devraient augmenter de $23 \%$. La contribution ordinaire de la Suisse atteindra 5,3 millions par an, ce qui correspond à 1,197\% du budget ordinaire de l'OMS.

La Convention-cadre pour la lutte antitabac est entrée en vigueur le 27 février $2005^{29}$. En décembre 2005, elle était signée par 158 Etats, dont la Suisse, et 114 pays l'avaient ratifiée ${ }^{30}$. Le secrétariat permanent de la convention aura son siège à Genève.

Annuaire 2005, $\mathrm{n}^{\circ}$ 1, Convention-cadre pour la lutte antitabac, pp. 132-133.

$2858^{\text {e }}$ Assemblée mondiale de la santé : <www.who.int/mediacentre/events/2005/wha58/fr>. Initiative «Pour un monde sans tabac»: <www.who.int/tobacco $>$ (en anglais seulement).

29 Convention-cadre de l'OMS pour la lutte antitabac: $<$ www.who.int/gb/fctc/F/F_index.htm $>$.

30 Statut de la Convention-cadre de l'OMS pour la lutte antitabac: <www.who.int/tobacco/framework/ countrylist/fr>. 
Du 7 au 9 novembre 2005, une conférence sur la grippe aviaire et la menace d'une pandémie de grippe humaine s'est tenue à Genève sous l'égide de l'ONU ${ }^{31}$. Elle a réuni plus de 600 représentants d'organisations internationales, de gouvernements et d'entreprises pharmaceutiques afin d'élaborer une stratégie de lutte contre la grippe aviaire. Selon le directeur général de l'OMS, Lee Jong Wook, il ne fait aucun doute que le virus responsable de la grippe aviaire va muter pour devenir transmissible entre humains; ce n'est qu'une question de temps.

$\mathrm{Au}$ terme de la conférence, les participants ont convenu d'un plan d'action visant à empêcher la propagation de l'infection. Ce plan prévoit notamment de vacciner les volailles d'élevage et d'améliorer le système d'alerte dans les pays en développement.

Le Rapport sur la santé dans le monde 2005, publié par l'OMS, est consacré aux taux de mortalité élevés parmi les femmes enceintes et les nourrissons que l'on enregistre dans les pays en développement ${ }^{32}$. Chaque année, environ un demi-million de femmes meurent pendant la grossesse ou en couches. Selon l'OMS, les principaux risques comprennent le manque d'hygiène, les maladies respiratoires ainsi que la diarrhée et le paludisme. Le rapport conclut que la vaccination, l'administration d'antibiotiques et un traitement administré par du personnel médical bien formé permettraient de sauver nombre de ces femmes. Par ailleurs, la prévention destinée à protéger la santé du nourrisson devrait commencer dès la grossesse. Il serait par exemple possible de réduire le nombre de décès parmi les jeunes enfants, si les nouveau-nés étaient «lavés, réchauffés et nourris » dans l'heure qui suit leur naissance. Ces gestes tout simples ont d'autant plus d'importance si l'on considère que 11 millions d'enfants meurent avant l'âge de 5 ans.

Un autre rapport sur la santé dans le monde a pour la première fois été publié en été 2005 33 . Intitulé Global Health Watch 2005-2006, il réunit des informations et des conclusions de divers mouvements en faveur de la santé, d'organisations non gouvernementales, de médecins et de scientifiques. Ce rapport dépeint la situation de la santé dans le monde du point de vue des personnes concernées, tant au Nord qu'au Sud, et analyse l'approche des organisations internationales de la santé.

\section{SOURCES}

Confédération suisse, $58^{e}$ Assemblée mondiale de la santé. Meilleure prévention contre les maladies infectieuses, communiqué de presse, 11 mai 2005.

Swissinfo, Grippe aviaire: Genève au cœur du combat, 7 novembre 2005 ; Mobilisation globale contre la grippe aviaire, 9 novembre 2005.

Neue Zürcher Zeitung, «Die Pandemie wird kommen», 7. November 2005.

\section{SITES INTERNET}

Organisation mondiale de la santé (OMS) : <www.who.int >.

Global Health Watch: <www.ghwatch.org >.

31 Conférence internationale sur la grippe aviaire et une pandémie de grippe humaine: <www.who.int/ mediacentre/events/2005/avian_influenza> (en anglais seulement).

32 Rapport sur la santé dans le monde 2005. Donnons sa chance à chaque mère et à chaque enfant, Genève, 2005, <www.who.int/whr/fr>.

33 Global Health Watch 2005-2006, New York, 2005, <www.ghwatch.org/2005_report.php>. 
La 93e session de la Conférence internationale du travail s'est tenue à Genève du 31 mai au 16 juin 2005 34 . Quelque 3000 délégués des gouvernements, des travailleurs et des employeurs des Etats membres de l'Organisation internationale du travail (OIT) y ont débattu de mesures destinées à résoudre des problèmes d'ordre social et liés à l'emploi. Parmi les principaux thèmes abordés, relevons l'élimination du travail forcé, les stratégies de lutte contre le chômage des jeunes et l'amélioration de la sécurité au travail.

Constatant que le recours au travail forcé persiste au Myanmar, la Commission de l'application des normes a invité les délégués à revoir leurs relations avec ce pays, en particulier dans les domaines des investissements directs, des sociétés publiques et de l'armée.

Les participants à la conférence ont adopté un programme et un budget de plus de 594 millions de dollars pour la période 2006-200735. Ce montant correspond à une hausse réelle de $1,1 \%$ du cadre financier. Le nouveau programme donne la priorité à la promotion d'un travail décent et prévoit des actions dans ce sens aux niveaux local, national, régional et international.

Le directeur général de l'OIT, Juan Somavia, a présenté à la conférence un rapport sur l'abolition du travail forcé, qui évalue l'importance économique régionale du travail forcé ${ }^{36}$ et propose un plan d'action pour l'abolir d'ici $2015^{37}$. L'OIT estime qu'au moins 12,3 millions de personnes dans le monde sont victimes du travail forcé. Deux tiers des cas sont recensés dans l'économie privée, un cinquième dans le secteur public ou militaire et un bon dixième dans l'exploitation commerciale du sexe. Les travailleurs forcés (dont les deux tiers vivent en Asie) ont réalisé un revenu annuel de quelque 32 milliards de dollars, dont près de la moitié provient de l'exploitation du sexe dans les pays industrialisés. L'objectif visant à abolir toutes les formes de travail forcé d'ici 2015 est considéré comme réaliste, puisque l'OIT entend impliquer d'autres organisations internationales dans cet effort. Une initiative concertée contre le travail forcé doit être lancée dès 2006.

Considérant le taux élevé du chômage parmi les jeunes, les délégués de plus de 100 pays ont recherché des moyens pour ouvrir des voies d'accès à un travail décent pour les jeunes. L'Organisation internationale du travail devra dès lors continuer à jouer un rôle important dans le Réseau pour l'emploi des jeunes (YEN, Youth Employment Network), afin d'étendre ce réseau à de nouveaux pays. Sachant que $85 \%$ des jeunes vivent dans des pays en développement, c'est surtout dans ces pays que l'offre d'emplois doit s'accroître.

34 Conférence internationale du travail : <www.ilo.org/public/french/standards/relm/ilc/ilc93>.

35 La contribution de la Suisse représente $1,198 \%$ du budget et se montera à 4,45 millions de francs par an.

36 Selon l'OIT, doit être considéré comme travail forcé ou obligatoire «tout travail ou service exigé d'un individu sous la menace d'une peine quelconque et pour lequel ledit individu ne s'est pas offert de son plein gré». Le travail forcé constitue alors une grave violation des droits de l'homme et une restriction de la liberté individuelle.

37 OIT, Une alliance mondiale contre le travail forcé. Rapport global en vertu du suivi de la Déclaration de l'OIT relative aux principes et droits fondamentaux au travail 2005, Genève, 2005. 
Répondant à l'appel du directeur général qui avait invité les délégués à présenter des réformes, la Suisse a soumis un vaste projet au plénum ${ }^{38}$. Elle a proposé de vérifier l'utilité des activités de l'OIT qui ont été décidées plus de cinq années auparavant et de renoncer aux tâches obsolètes. La Suisse a par ailleurs plaidé en faveur d'une reconsidération de l'efficacité des travaux de la Commission des résolutions, politiquement contestée. Enfin, elle a suggéré d'améliorer l'efficacité de la Conférence du travail: l'assemblée plénière devrait désormais se tenir tous les deux ans en alternance avec une conférence technique. Ces diverses mesures permettraient de gagner du temps et allégeraient la charge financière de l'OIT et de ses conférences.

\section{SOURCES}

Organisation internationale du travail (OIT), Travail, le magazine de l'OIT, nº 54, août 2005.

Confédération suisse, 93e session de la Conférence internationale du travail, communiqué de presse, 4 mai 2005; Ouverture de la procédure de consultation relative à l'avant-projet de rapport «La situation des gens du voyage en Suisse », communiqué de presse, 22 juin 2005.

Le Temps, «Travail forcé: 12,3 millions de victimes», 12 mai 2005.

Neue Zürcher Zeitung, «Weltweiter Kampf gegen die Zwangsarbeit», 13. Mai 2005; «Die ILO auf der Suche nach einem neuen Stil», 1. Juni 2005.

\section{SITE INTERNET}

Organisation internationale du travail (OIT) : <www.ilo.org $>$.

\subsection{UNESCO}

Organisation des Nations unies pour l'éducation, la science et la culture

\subsubsection{Adoption de la Convention internationale sur la protection et la promotion de la diversité des expressions culturelles}

Le 20 octobre 2005, la Conférence générale de l'UNESCO a approuvé, par 148 voix contre 2 (Etats-Unis et Israël) et 4 abstentions (Australie, Liberia, Honduras et Nicaragua), la Convention sur la protection et la promotion de la diversité des expressions culturelles, surnommée plus sobrement convention pour la diversité culturelle ${ }^{39}$. Cet instrument juridique international entrera en vigueur trois mois après sa ratification par au moins 30 des 191 Etats membres de l'UNESCO. Mais, d'un point de vue politique et pour donner sa pleine portée à la convention, il est nécessaire que 50 à 60 pays la ratifient au cours des deux à trois prochaines années, et ce dans toutes les régions du monde: l'Afrique, l'Asie-Océanie, les Amériques et l'Europe.

Un avant-projet élaboré par un groupe d'experts a servi de base de discussion et d'intenses négociations. Deux années, jalonnées de plusieurs réunions d'experts indépendants, puis gouvernementaux, ont été nécessaires pour aboutir au texte final, qui reprend les grands principes formulés dans la Déclaration universelle de l'UNESCO sur la diversité culturelle, adoptée à l'unanimité en 2001: la diversité culturelle doit être considérée comme un «patrimoine commun de

38 «Glanzlose ILO-Jahreskonferenz», Neue Zürcher Zeitung, 16. Juni 2005.

39 Le texte de la convention est disponible sur la page Internet de l'UNESCO consacrée à la culture : $<$ http://portal.unesco.org/culture/fr>. 
l'humanité» et sa «défense comme un impératif éthique, inséparable du respect de la dignité de la personne humaine».

An Annuaire 2005, $\mathrm{n}^{\circ}$ 1, processus de négociation et présentation des enjeux de la Convention sur la diversité culturelle, pp. 139-142.

L'ultime round de négociations, qui s'est tenu en octobre 2005 lors de la $33^{\circ}$ Conférence générale de l'UNESCO, a été marqué par la position des Etats-Unis, qui cherchaient à bloquer le processus d'adoption. Les Etats-Unis visaient à soumettre le commerce des biens culturels aux règles de l'Organisation internationale du commerce (OMC), notamment en interdisant les subventions publiques comme soutien à la production artistique, considérées comme «une distorsion de concurrence». Mais malgré le puissant lobby mis en œuvre par les Etats-Unis, l'adoption de la convention a pu être obtenue à une très large majoritét ${ }^{40}$.

Parmi les nombreux principes établis dans la convention, quelques éléments fondamentaux méritent d'être relevés. En matière de diversité culturelle, la convention pose, comme postulat de départ, le principe du respect des droits de l'homme et des libertés fondamentales, notamment en matière de liberté d'expression, d'information et de communication, ainsi que, pour les individus, le choix de leurs expressions culturelles. Le texte admet la double nature des biens et services culturels, à la fois vecteurs essentiels de la diffusion des œuvres artistiques (porteurs d'identité, de valeurs et de sens) et objets de commerce (valeur économique). Il est reconnu un droit souverain, pour les Etats, d'adopter par le biais de politiques culturelles des mesures pour la promotion et la protection de la diversité des expressions culturelles, y compris la diversité des médias. La diversité culturelle est considérée comme un facteur fondamental du développement durable, notamment dans les pays en voie de développement. La convention vise à réaffirmer les liens qui unissent culture, développement et dialogue et à créer une plate-forme innovante de coopération culturelle internationale. Pour atteindre ces différents objectifs, il apparaît indispensable de donner à la diversité culturelle une place dans l'ordre juridique international, en assurant l'égalité entre la convention et les autres instruments internationaux (soutien mutuel, complémentarité et non-subordination). En outre, un rôle important est reconnu à la société civile dans la mise en place de la Convention ${ }^{41}$.

\section{$\square$ Position de la Suisse}

Comme le souligne l'Office fédéral de la culture (OFC), la diversité culturelle constitue un enjeu majeur pour la Suisse; de plus, elle est ancrée dans la Constitution fédérale ${ }^{42}$. Cela explique pourquoi la Suisse a soutenu activement le processus d'élaboration de la convention dès le début et a apporté une contribution importante dans les négociations. Sur le fond, la Suisse a notamment

40 «La Convention sur la diversité culturelle en débat à l'UNESCO», Le Monde, 15 octobre 2005. «UNESCO-Konvention über kulturelle Vielfalt. Protektionismus gegen Amerikas Vormachtstellung», Neue Zürcher Zeitung, 19. Oktober 2005. «UNESCO-Votum gegen Amerika», Neue Zürcher Zeitung, 22. Oktober 2005.

41 UNESCO, La Conférence générale adopte la Convention sur la protection et la promotion de la diversité des expressions culturelles, communiqué de presse, 20 octobre 2005 (présentation résumée des principaux articles de la convention).

42 OFC, position de la Suisse sur la diversité culturelle, <www.bak.admin.ch/bak/index.html?lang=fr> >thèmes >politique culturelle >diversité culturelle. 
défendu deux exigences fondamentales: la pluralité des médias et l'implication de la société civile, en particulier des ONG, dans la mise en œuvre de la diver-

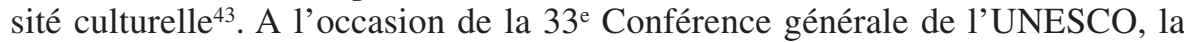
Suisse s'est clairement exprimée en faveur de l'adoption de la convention, pour laquelle elle lancera la procédure de ratification vraisemblablement en 2006.

Cependant, le secteur culturel de la société civile, réuni dans la Coalition suisse pour la diversité culturelle (voir plus bas), relève que le processus de ratification se fera parallèlement aux négociations de l'accord de libre-échange entre la Suisse et les Etats-Unis agendées pour 2006 et pense que les Etats-Unis insisteront pour inclure le secteur de la culture et de la communication digitale dans cet accord. Ainsi, la Coalition suisse pour la diversité culturelle anticipe certaines divergences de vues entre les responsables fédéraux de la culture et ceux du Secrétariat d'Etat à l'économie (seco) en charge de la négociation de l'accord bilatéral.

\section{$\square$ Coalitions nationales pour la diversité culturelle}

Les organisations de la culture se sont organisées sur le plan international en «coalitions nationales pour la diversité culturelle». La coalition suisse regroupe près de 70 organisations ${ }^{44}$. Les 31 coalitions ainsi constituées rassemblent plus de 500 organisations professionnelles de la culture, représentant des écrivains, des compositeurs, des réalisateurs, des artistes de la scène et des arts plastiques, des éditeurs, producteurs et distributeurs indépendants de films, de musique et d'émissions de télévision, ainsi que des sociétés publiques de radiodiffusion. Elles ont mené une active campagne en faveur de l'adoption de la convention et s'engagent maintenant pour une ratification rapide afin que la convention puisse entrer en vigueur le plus tôt possible.

Dans un communiqué de presse, les coalitions ont souligné que «le vote très largement majoritaire en faveur de la convention indique que le droit d'adopter des politiques culturelles (quotas sur le contenu national, subventions, crédits d'impôt, règles sur la propriété étrangère, etc.) est désormais reconnu comme un droit primordial par les pays à travers le monde. Et ce pour une raison fort simple: à de très rares exceptions près, les pays ont besoin de pouvoir recourir à de telles mesures pour assurer à leurs citoyens et citoyennes l'accès à leur propre culture. Des niveaux substantiels de production culturelle nationale sont essentiels pour qu'il y ait un échange international équilibré de biens et services culturels entre tous les pays du monde. La pression croissante que les négociations commerciales exercent sur les pays pour qu'ils renoncent à leur droit d'adopter des politiques culturelles rendait impérieuse l'adoption de la convention de l'UNESCO dans les plus brefs délais $»^{45}$.

\section{Evaluation du commerce des biens culturels}

43 Y. Jaggi, «UNESCO contre OMC: deux visions de la culture dans le monde s'affrontent », Le Temps, rubrique «Eclairages », 23 juin 2005.

44 Site Internet: <www.coalitionsuisse.ch>.

45 Comité international de liaison des coalitions, communiqué de presse, Paris, 21 octobre 2005. 
En décembre 2005, I'Institut de statistique de I'UNESCO (ISU) a publié son premier rapport consacré au commerce de biens culturels, qui analyse les données du commerce international de 120 pays pour une sélection de biens et services culturels: médias imprimés (livres, journaux), médias enregistrés (disques, bandes magnétiques sonores), arts visuels (peintures, gravures, sculptures) et médias audiovisuels (dominés par les jeux vidéo, DVD).

Le poids économique du secteur des industries culturelles et créatives est estimé à 1,3 billion de dollars (soit $7 \%$ du PIB mondial) et progresse rapidement. Selon le rapport, le commerce international des biens culturels est passé de 38 milliards à 60 milliards de dollars entre 1994 et 2002. L'Amérique latine et les Caraïbes n'ont représenté que $3 \%$ du commerce international des biens culturels en 2002, à peine un point de plus qu'en 1992. L'Océanie et l'Afrique n'ont pas enregistré de progrès, totalisant ensemble un peu moins de $1 \%$. En 2002, les 15 pays de I'Union européenne dominaient les exportations de biens culturels, affichant une part de 51,8\%. L'Asie s'est avérée la deuxième région exportatrice avec une part de 20,6\% du total des exportations mondiales. S'ensuit l'Amérique du Nord, passée au troisième rang des régions exportatrices, régressant de $25 \%$ en 1994 à 16,9\% en 2002 . Mais ces chiffres régionaux cachent une autre réalité: trois pays (Royaume-Uni, Etats-Unis et Chine) représentaient $40 \%$ du commerce mondial des biens culturels.

Les auteurs du rapport ont utilisé une nouvelle méthodologie afin de mieux refléter les flux du commerce culturel, mais ils reconnaissent certaines limites à l'exercice. Ces efforts seront poursuivis dans les années à venir, en vue notamment de fournir des données plus précises et de répondre ainsi à une des exigences de la Convention sur la diversité culturelle adoptée en octobre 2005.

Sources: Institut de statistique de I'UNESCO, Echanges internationaux d'une sélection de biens et services culturels, 19942003. Définir et évaluer le flux du commerce culturel mondial, Montréal, ISU, 2005. UNESCO, Les pays en développement sont les perdants du commerce des biens culturels, communiqué de presse, 15 décembre 2005.

\section{SOURCES}

UNESCO, Convention sur la protection et la promotion de la diversité des expressions culturelles, adoptée lors de la 33 Conférence générale de l'UNESCO, Paris, 20 octobre 2005.

Bernier I. en collaboration avec Ruiz Fabri H., La mise en œuvre et le suivi de la Convention de l'UNESCO sur la protection et la promotion de la diversité des expressions culturelles, s.d., disponible sur Internet: <www.mcc.gouv.qc.ca/international/diversite-culturelle/mise_en_oeuvre_unesco.htm>.

Déclaration de Berne, Vers un développement solidaire, dossier «Diversité culturelle», $\mathrm{n}^{\circ} 182$, octobre 2005.

Le Monde, «La Convention sur la diversité culturelle en débat à l’UNESCO», 15 octobre 2005.

Le Monde diplomatique, A. Mattelard, «Des biens et des services porteurs d'identité, de valeurs et de sens. Bataille à l'UNESCO sur la diversité culturelle», octobre 2005.

Le Temps, «Diversité culturelle, une bataille planétaire», 21 octobre 2005; Y. Jaggi, «UNESCO contre OMC: deux visions de la culture dans le monde s'affrontent», rubrique «Eclairages», 23 juin 2005.

Neue Zürcher Zeitung, «UNESCO-Konvention über kulturelle Vielfalt. Protektionismus gegen Amerikas Vormachtstellung», 19. Oktober 2005 ; «UNESCO-Votum gegen Amerika », 22. Oktober 2005.

\section{SITES INTERNET}

UNESCO: <www.unesco.org>.

UNESCO, page consacrée à la culture : <http://portal.unesco.org/culture/fr> .

Commission suisse pour l'UNESCO : <www.unesco.ch>.

Commission suisse pour l'UNESCO, page consacrée à la diversité culturelle : <www.unesco.ch/work-f/ diversite.htm>.

Coalition suisse pour la diversité culturelle: <www.coalitionsuisse.ch>. 
Lors de sa session de septembre 2005, l'Assemblée générale de l'ONU a voté la dissolution de la Commission des droits de l'homme et la création d'un Conseil des droits de l'homme. La Suisse et les autres partisans du projet devront à présent veiller à ce que le conseil soit doté de structures qui empêcheront sa politisation et combleront les lacunes de l'actuelle commission.

Pour la première fois de son existence, la Cour pénale internationale a lancé des mandats d'arrêt. La cour ne disposant cependant d'aucun moyen d'exécution, on ne sait pas s'ils suffiront pour faire arrêter les personnes recherchées.

\subsubsection{Session de la Commission des droits de l'homme}

La $61^{\mathrm{e}}$ session de la Commission des droits de l'homme de l'ONU s'est tenue à Genève du 14 mars au 22 avril 2005. L'organe a siégé pendant six semaines et adopté 86 résolutions, 16 décisions et 4 déclarations ${ }^{46}$. La commission a créé trois nouveaux mandats: expert indépendant sur les questions relatives aux minorités, expert indépendant sur les droits de l'homme et la solidarité internationale; et représentant spécial chargé de la question des droits de l'homme et des sociétés internationales.

Pendant la session, le secrétaire général des Nations unies, Kofi Annan, a par ailleurs présenté sa proposition de créer un nouveau Conseil des droits de l'homme, pour remplacer l'actuelle commission (voir plus bas section 9.5.2) (7) $^{4}$. Divers Etats et des ONG ont à nouveau critiqué l'ingérence de la politique dans les travaux de la commission, et même la commission d'experts pour la réforme des Nations unies, instituée par Kofi Annan, a constaté l'incapacité de la commission à faire respecter les droits de l'homme dans le monde entier ${ }^{48}$.

Le thème «terrorisme et droits de l'homme» a occupé une large place dans les débats de la session et la commission a fini par nommer un expert indépendant sur la protection des droits de l'homme et des libertés fondamentales dans la lutte antiterroriste. Dans les différents textes adoptés sur le terrorisme, la commission appelle les Etats à ne pas faire de la lutte contre le terrorisme un prétexte pour limiter la liberté de parole. Elle a également exigé que les mesures prises par les Etats pour combattre le terrorisme n'entravent pas le travail des défenseurs des droits de l'homme et ne menacent pas la sécurité. La commission s'est par ailleurs déclarée "profondément préoccupée par le fait que l'islam est souvent et faussement associé aux vioations des droits de l'homme et au terrorisme».

Pour ce qui est de la situation des droits de l'homme pays par pays, la commission a condamné les violations de ces droits dans les Etats suivants: Myanmar, Corée du Nord, Cuba, Bélarus et Soudan. Une résolution présentée par Cuba sur les droits des détenus à la bases militaire américaine de Guantanamo a été rejetée.

46 Commission des droits de l'homme, Rapport sur la 61 e session, Documents officiels, 2005, Supplément $\mathrm{n}^{\mathrm{o}}$ 3, New York; Genève, 2005.

47 «Kofi Annan plaide pour que la situation des droits de l'homme soit passée au crible dans tous les pays », Le Temps, 8 avril 2005.

48 Swissinfo, Pour défendre réellement les droits de l'homme, 7 avril 2005. 
Sur l'initiative de la Suisse, la commission a adopté une résolution sur le Népal. Celle-ci appelle le gouvernement népalais à rétablir la démocratie, les droits civiques et les droits politiques, et à mettre fin aux exécutions extrajudiciaires, à la violence sexuelle et aux détentions arbitraires ${ }^{49}$. La résolution condamne aussi les pratiques des maoïstes népalais, notamment les exécutions sommaires, les actes de persécution, les enlèvements et le travail forcé. Relevons que le gouvernement népalais avait accepté, deux semaines auparavant, l'ouverture d'un bureau des droits de l'homme de l'ONU dans sa capitale, Katmandou. C'est cette concession qui aurait motivé la Suisse à adoucir la formulation de son projet de résolution, initialement plus tranchante.

Le 20 mai 2005, la haut-commissaire aux droits de l'homme, Louise Arbour, a présenté, comme prévu, un plan d'action destiné à améliorer le travail du HautCommissariat aux droits de l'homme ${ }^{50}$. Ce document stratégique identifie les lacunes dont souffre la promotion des droits de l'homme et émet des propositions destinées à réduire le clivage entre la rhétorique et la réalité dans ce domaine. La haut-commissaire espère par ailleurs pouvoir déléguer un plus grand nombre d'observateurs sur place, afin de mener des activités de maintien de la paix. Elle juge également important de pouvoir répondre rapidement aux demandes d'aide des Etats.

Pour mettre en œuvre le plan d'action, il faudrait cependant doubler les ressources financières et les effectifs du haut-commissariat dans les cinq années à venir. En 2004, son budget atteignait 83 millions de dollars, dont seuls 34 millions provenaient du budget ordinaire de l'ONU (soit 1,8\% de son budget ordinaire). En conséquence, la majeure partie du financement est assurée par des contributions volontaires. La même année, les effectifs se montaient à 580 personnes, dont 310 collaborateurs basés à Genève.

\subsubsection{Création d'un Conseil des droits de l'homme}

Le 21 mars 2005, le secrétaire général des Nations unies, Kofi Annan, a présenté le rapport intitulé Dans une liberté plus grande: développement, sécurité et respect des droits de l'homme pour tous, qui préconise de profondes réformes de l'ONU ${ }^{51}$. Ce document propose aussi un modèle d'organisation pour un Conseil des droits de l'homme. Le projet s'inspire d'une initiative de la Suisse et vise à remplacer la Commission des droits de l'homme, frappée de discrédit, par un Conseil des droits de l'homme doté d'un statut supérieur.

[D] Annuaire 2005, $\mathrm{n}^{\circ}$ 1, Commission des droits de l'homme, pp. 144-148.

Le Conseil des droits de l'homme prévu par Kofi Annan doit compter 24 membres, élus par l'Assemblée générale à la majorité des deux tiers, et l'une de ses principales tâches devrait consister à vérifier régulièrement si tous les Etats respectent leurs obligations en matière de droits de l'homme. En cas de crise, le conseil devrait pouvoir se réunir rapidement pour prendre des décisions. Il

49 Swissinfo, Sur le Népal, l'ONU suit la Suisse, 20 avril 2005.

50 «Human Rights Commission Holds 61st Session amidst Debate on Reform», Go Between, $\mathrm{n}^{\circ}$ 107, April-May-June 2005.

51 Rapport du secrétaire général de l'ONU à l'Assemblée générale à l'occasion du Sommet mondial en septembre 2005, Dans une liberté plus grande: développement, sécurité et respect des droits de l'homme pour tous, doc. A/59/2005, New York, 21 mars 2005, <www.un.org/french/largerfreedom>. 
devrait dès lors travailler en permanence et non pas seulement six semaines par année comme le fait la commission.

Nombre de pays du Sud s'opposent à la diminution du nombre des membres et à l'adoption de conditions restreignant l'éligibilité au sein du conseil. A l'inverse, nombre de pays occidentaux appuient les propositions de réforme de Kofi Annan, notamment celle prévoyant que le conseil siège en permanence, du moins plusieurs fois par année.

Les représentants des organisations non gouvernementales craignent que la réforme remette en cause leur droit de consultation. Ils souhaitent par ailleurs que le conseil reprenne à son compte les mécanismes efficaces mis en place par la commission, tel l'envoi d'experts chargés d'évaluer le respect des droits de l'homme dans certains pays ${ }^{52}$.

En 2005, la Suisse a défendu énergiquement l'idée de créer un Conseil des droits de l'homme. Elle peut d'ailleurs compter sur deux alliés de poids: les Etats-Unis et le président de la $60^{\mathrm{e}}$ session de l'Assemblée générale, le Suédois Jan Eliasson ${ }^{53}$. Que ce soit à l'occasion de la session de la Commission des droits de l'homme ou de la $60^{\text {e }}$ session de l'Assemblée générale de l'ONU, la conseillère fédérale Micheline Calmy-Rey et le conseiller fédéral Samuel Schmid ont plaidé pour une mise sur pied rapide de ce conseil.

Pour la Suisse, il importe surtout que le conseil jouisse d'une position élevée dans l'organigramme des Nations unies (qu'il figure parmi les principaux organes ou qu'il soit directement subordonné à l'Assemblée générale); qu'il se réunisse périodiquement; qu'il soit sis à Genève et qu'il collabore étroitement avec le Haut-Commissariat aux droits de l'homme. La taille du conseil devrait garantir sa légitimité et une bonne représentativité, sans entraver son efficacité. La Suisse préconise par ailleurs de reprendre les instruments de la commission qui ont jusqu'ici fait leurs preuves ${ }^{54}$.

La création d'un Conseil des droits de l'homme figure dans la déclaration finale du débat sur la réforme de l'ONU qui a eu lieu pendant l'Assemblée générale, en septembre 2005. Le rôle du futur conseil y est défini en ces termes: «Le conseil sera chargé de promouvoir le respect universel et la défense de tous les droits de l'homme et de toutes les libertés fondamentales, pour tous, sans aucune sorte de distinction et de façon juste et équitable. $\gg^{55}$ Cette description ne fournit aucune indication sur le mode de fonctionnement, la composition, les critères d'élection et le siège du conseil. Le Sommet mondial a chargé l'Assemblée générale de régler ces différents points.

En septembre 2005, 20 Etats, dont les poids lourds que sont la Russie et la Chine, étaient fermement opposés au projet de créer un Conseil des droits de l'homme. Le secrétaire général prévoit néanmoins que le conseil sera opérationnel dès le mois de mars 2006 et qu'il aura son siège à Genève ${ }^{56}$.

52 Pour connaître les détails de la positions des organisations de défense des droits de l'homme, voir: Fédération internationale des ligues des droits de l'homme (FIDH), Conseil des droits de l'homme. Renforcer la protection, octobre 2005, <www.fidh.org >.

53 «Schweiz investiert in UNO-Reform», Tagesanzeiger, 21. September 2005.

54 Peter Maurer, Déclaration lors de la $59^{\mathrm{e}}$ session de l'Assemblée générale, «Cluster III : Vivre dans la dignité», New York, 19 avril 2005.

55 Document final du Sommet mondial de 2005, résolution adoptée par l'Assemblée générale, doc. A/RES/60/1, New York, 24 octobre 2005.

56 «Menschenrechtsrat nach Genf», Tagesanzeiger, 7. Oktober 2005. 


\subsubsection{Travail de la Cour pénale internationale}

La Cour pénale internationale (CPI) est issue du Statut de Rome, adopté en 1998 et ratifié par 99 Etats jusqu'en octobre $2005^{57}$. Indépendante de l'ONU et sise à La Haye, cette institution est compétente pour juger les cas de génocide, de crime contre l'humanité et de crime de guerre, pour autant que l'Etat concerné n'ait pas la volonté ou la possibilité de mener lui-même l'instruction et la procédure pénales.

Jusqu'en octobre 2005, le procureur de la CPI avait ouvert des instructions dans trois pays: la République démocratique du Congo, l'Ouganda et le Soudan. L'ouverture de bureaux locaux à Kinshasa et à Bunia (République démocratique du Congo) et à Kampala (Ouganda) devrait faciliter le travail de la cour et assurer une certaine protection aux témoins et aux victimes.

Une instruction a pu être ouverte au Soudan suite à l'adoption d'une résolution par le Conseil de sécurité de l'ONU, qui a confié, le 31 mars 2005, le cas du Darfour $^{58}$ à la CPI. C'est la première fois que ce conseil a transmis un cas à la cour et la résolution en question a été adoptée par 11 voix contre 0 lors des délibérations. Même les Etats-Unis, qui ne reconnaissent pourtant pas la CPI, se sont abstenus. Soulignons toutefois qu'ils avaient au préalable reçu l'assurance que les citoyens américains travaillant au Soudan ne seraient pas concernés par la résolution.

En octobre 2005, la Cour pénale internationale a lancé ses premiers mandats d'arrêt contre cinq dirigeants du groupe rebelle ougandais Lord's Resistance Army (LRA) et indiqué aux gouvernements ougandais, soudanais et congolais les lieux où les suspects séjournaient vraisemblablement ${ }^{59}$. Le Statut de Rome n'accordant aucun moyen d'exécution (des forces de police, p. ex.) à la cour pénale, l'efficacité de celle-ci repose entièrement sur la bonne volonté des Etats qui la soutiennent.

En ce qui concerne la Suisse, le Conseil fédéral entend réviser la législation pour adapter le droit pénal matériel au Statut de Rome instituant la Cour pénale internationale ${ }^{60}$. En août 2005, il a soumis les modifications nécessaires à une procédure de consultation. Ces modifications introduisent la notion de crime contre l'humanité dans la législation fédérale. De plus, des crimes tels que les actes de guerre contre la population civile ou le recours à des armes interdites figureront à l'avenir expressément dans le code pénal.

Le projet prévoit que les cas de crime contre l'humanité ou de génocide ne seront poursuivis que si leur auteur possède un «lien étroit» avec la Suisse. Cette restriction a notamment été critiquée par l'Association suisse contre l'impunité (TRIAL).

57 Les Etats-Unis, l'Inde, la Chine et la Russie n'ont pas ratifié le Statut de Rome.

58 Une milice arabe bénéficiant de l'appui du gouvernement est accusée de graves violations des droits de l'homme sur la population non arabe du Darfour.

59 La LRA opère depuis bientôt vingt ans dans le nord de l'Ouganda et se compose principalement d'enfants, enrôlés de force ou réduits à l'esclavage sexuel. Aux yeux du coordinateur de l'aide humanitaire de l'ONU, Jan Egeland, c'est «le groupe rebelle le plus brutal au monde». «Friedenshoffnungen in Norduganda», Neue Zürcher Zeitung, 28. September 2005. «Haager Strafgericht will Ugander aburteilen», Neue Zürcher Zeitung, 8. Oktober 2005.

60 La notion de génocide a été introduite dans le droit suisse en 2000 déjà. «Hüst und Hott gegen schwerste Verbrechen», Tagesanzeiger, 18. August 2005. 
Le 16 décembre 2004, diverses ONG qui militent en faveur d'une Cour pénale internationale au fonctionnement efficace ont fondé la Coalition suisse pour la Cour pénale internationale ${ }^{61}$. La coalition s'engage en faveur d'un droit pénal international fort et pour la poursuite effective des responsables de crimes internationaux tels que les génocides, les crimes de guerre, les crimes contre l'humanité et la torture. Elle entend notamment mener des actions pour favoriser l'intégration du crime contre l'humanité dans le droit pénal suisse et créer les conditions requises pour que les crimes de guerre soient effectivement poursuivis en Suisse.

En juin 2005, la coalition a demandé au Conseil fédéral de respecter ses engagements internationaux dans la lutte contre le commerce illégal de ressources naturelles exploitées au Congo. En menant des activités au Congo, des entreprises commerciales sises en Suisse soutiennent en effet divers groupes armés sur place, qui disposent ainsi d'une source de financement et perpétuent le conflit armé.

Annuaire 2006, $\mathrm{n}^{\circ}$ 1, droits de l'homme et politique suisse de l'asile: voir chap. 12, «Politique d'asile et questions migratoires ».

\section{SOURCES}

Assemblée générale des Nations unies, Document final du sommet mondial de 2005, résolution adoptée par l'Assemblée générale, doc. A/RES/60/1, New York, 24 octobre 2005.

Calmy-Rey, Micheline, Déclaration lors de la $60^{\circ}$ session de l'Assemblée générale de l'ONU, 20 septembre 2005.

Calmy-Rey, Micheline, Déclaration lors de la $61^{\mathrm{e}}$ session de la Commission des droits de l'homme de l'ONU, 14 mars 2005.

Département fédéral de justice et police, Crimes contre l'humanité et crimes de guerre font leur entrée dans le code pénal, communiqué de presse, 17 août 2005.

Le Temps, «Droits humains: Micheline Calmy-Rey fière de ses diplomates», 22 septembre 2005.

Neue Zürcher Zeitung, «Rat oder Kommission für Menschenrechte», 15. April 2005.

\section{SITES INTERNET}

Commission des droits de l'homme de l'ONU: <www.ohchr.org/french/bodies/chr>.

Cour pénale internationale (CPI) : <www.icc-cpi.int $>$.

Département fédéral de justice et police : <www.ejpd.admin.ch $>$.

Association suisse contre l'impunité (TRIAL) : $<w w w . t r i a l-c h . o r g ~>$.

Coalition des ONG pour la Cour pénale internationale: $<w w w . i c c n o w . o r g>$.

Coalition suisse pour la Cour pénale internationale: $<w w w . t r i a l-c h . o r g / c o a l i t i o n>$.

humanrights.ch (Association suisse pour les droits de 1'homme) : <www.humanrights.ch $>$.

Human Rights Watch: <www.hrw.org/french>.

61 Voici les membres fondateurs de la coalition: Action des chrétiens pour l'abolition de la torture (ACAT), Association pour la prévention de la torture (APT), Amnesty International - Section suisse, Société pour les peuples menacés (SPM), humanrights.ch (Association suisse pour les droits de la personne), Alliance Sud, Association suisse contre l'impunité (TRIAL) et Fédéralistes mondiaux Suisse. 